Marquette University

e-Publications@Marquette

12-3-2003

\title{
The dapE-encoded N-Succinyl-I,I-Diaminopimelic Acid Desuccinylase from Haemophilus influenzae Is a Dinuclear Metallohydrolase
}

\author{
Nathaniel J. Cosper \\ University of Georgia \\ David L. Bienvenue \\ Utah State University \\ Jacob E. Shokes \\ University of Georgia \\ Danuta M. Gilner \\ Utah State University \\ Takashi Tsukamoto \\ Guilford Pharmaceuticals Inc.
}

See next page for additional authors

Follow this and additional works at: https://epublications.marquette.edu/chem_fac

Part of the Chemistry Commons

\section{Recommended Citation}

Cosper, Nathaniel J.; Bienvenue, David L.; Shokes, Jacob E.; Gilner, Danuta M.; Tsukamoto, Takashi; Scott, Robert A.; and Holz, Richard C., "The dapE-encoded N-Succinyl-I,I-Diaminopimelic Acid Desuccinylase from Haemophilus influenzae Is a Dinuclear Metallohydrolase" (2003). Chemistry Faculty Research and Publications. 314.

https://epublications.marquette.edu/chem_fac/314 


\section{Authors}

Nathaniel J. Cosper, David L. Bienvenue, Jacob E. Shokes, Danuta M. Gilner, Takashi Tsukamoto, Robert A. Scott, and Richard C. Holz 
Marquette University

\title{
e-Publications@Marquette
}

\section{Chemistry Faculty Research and Publications/College of Arts and Sciences}

This paper is NOT THE PUBLISHED VERSION; but the author's final, peer-reviewed manuscript. The published version may be accessed by following the link in the citation below.

Journal of the American Chemical Society, Vol. 125, No. 48 (November 5, 2003): 14654-14655. DOI. This article is (C) American Chemical Society Publications and permission has been granted for this version to appear in e-Publications@Marquette. American Chemical Society Publications does not grant permission for this article to be further copied/distributed or hosted elsewhere without the express permission from American Chemical Society Publications.

\section{The dapE-encoded $\mathrm{N}$-Succinyl-I,I- Diaminopimelic Acid Desuccinylase from Haemophilus influenzae Is a Dinuclear Metallohydrolase}

\author{
Nathaniel J. Cosper \\ Department of Chemistry, University of Georgia, Athens, Georgia \\ David L. Bienvenue \\ Department of Chemistry and Biochemistry, Utah State University, Logan, Utah \\ Jacob E. Shokes \\ Department of Chemistry, University of Georgia, Athens, Georgia \\ Danuta M. Gilner \\ Department of Chemistry and Biochemistry, Utah State University, Logan, Utah \\ Guilford Pharmaceuticals Inc., 6611 Tributary Street, Baltimore, Maryland \\ Takashi Tsukamoto \\ Guilford Pharmaceuticals Inc., 6611 Tributary Street, Baltimore, Maryland
}




\section{Robert A. Scott}

Department of Chemistry, University of Georgia, Athens, Georgia

Richard C. Holz

Department of Chemistry and Biochemistry, Utah State University, Logan, Utah

\section{SUBJECTS:}

Infectious diseases, Peptides and proteins, lons

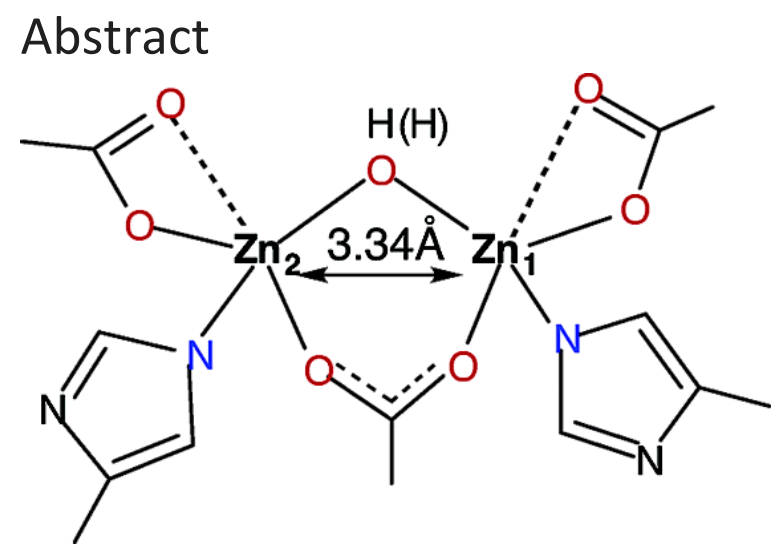

The Zn K-edge extended X-ray absorption fine structure (EXAFS) spectra, of the dapE-encoded $\mathrm{N}$-succinyl-I,Idiaminopimelic acid desuccinylase (DapE) from Haemophilus influenzae have been recorded in the presence of one or two equivalents of Zn(II) (i.e. [Zn_(DapE)] and [ZnZn(DapE)]). The Fourier transforms of the Zn EXAFS are dominated by a peak at ca. 2.0 $\AA$, which can be fit for both [Zn_(DapE)] and [ZnZn(DapE)], assuming ca. $5(\mathrm{~N}, \mathrm{O})$ scatterers at 1.96 and $1.98 \AA$, respectively. A second-shell feature at ca. $3.34 \AA$ appears in the [ZnZn(DapE)] EXAFS spectrum but is significantly diminished in [Zn_(DapE)]. These data show that DapE contains a dinuclear $\mathrm{Zn}$ (II) active site. Since no X-ray crystallographic data are available for any DapE enzyme, these data provide the first glimpse at the active site of DapE enzymes. In addition, the EXAFS data for DapE incubated with two competitive inhibitors, 2-carboxyethylphosphonic acid and 5-mercaptopentanoic acid, are also presented.

According to recent estimates from the United States Centers for Disease Control and Prevention (the CDC), more than 10 million people died worldwide from bacterial infections in $1995 .{ }^{1}$ Tuberculosis, for example, was the leading cause of death in adults by an infectious disease. ${ }^{2}$ The importance of developing new drugs to fight infectious disease, such as tuberculosis, is underscored by the emergence of several pathogenic bacterial strains that are resistant to all currently available antibiotics. ${ }^{3-6}$ To overcome bacterial resistance to antibiotics, new enzyme targets must be located and small-molecule inhibitors developed. The meso-diaminopimelate (mDAP)/lysine biosynthetic pathway offers several potential anti-bacterial targets that have yet to be explored. ${ }^{7-}$ ${ }^{9}$ Since both products of this pathway, mDAP and lysine, are essential components of the peptidoglycan cell wall in Gram-negative and some Gram-positive bacteria, inhibitors of enzymes within this pathway may provide a new class of antibiotics. ${ }^{5}$ The fact that there are no similar pathways in mammals suggests that inhibitors of enzymes in the mDAP/lysine pathway will provide selective toxicity against bacteria and will potentially have little or no effect on humans.

One of the enzymes in this pathway, ${ }^{10}$ the $d a p E$-encoded $N$-succinyl-I,I-diaminopimelic acid desuccinylase (DapE), catalyzes the hydrolysis of $\mathrm{N}$-succinyl-I,I-diaminopimelate to I,I-diaminopimelate and succinate. ${ }^{11}$ It has 
been shown that deletion of the gene encoding DapE is lethal to Helicobacter pylori and Mycobacterium smegmatis. ${ }^{12,13}$ Even in the presence of lysine-supplemented media, the DapE deletion cell line of H. pylori was unable to grow. Therefore, DapEs are essential for cell growth and proliferation. Since no structural data of any kind are available for DapE enzymes, we have recorded the Zn K-edge extended X-ray absorption fine structure (EXAFS) spectra of the DapE from $H$. influenzae in the presence of one or two equivalents of $Z n$ (II) (i.e.

[Zn_(DapE)] and [ZnZn(DapE)]) (Figure 1). For [ZnZn(DapE)] the EXAFS data report an average of both metal ion environments. The Fourier transforms of both [Zn_(DapE)] and [ZnZn(DapE)] are dominated by a peak at ca. 2.0 $\AA$, that can be fit for both [Zn_(DapE)] and [ZnZn(DapE)] assuming $5(\mathrm{~N}, 0)$ scatterers, at 1.96 and $1.98 \AA$, respectively (Fits 1,3; Table 1). A second shell feature at ca. $3.3 \AA$ appears in the [ZnZn(DapE)] EXAFS spectrum but is significantly diminished in [Zn_(DapE)]. Fits incorporating a Zn-Zn interaction at $3.34 \AA$ for [ZnZn(DapE)] have reasonable Debye-Waller factors (Fit 4; Table 1) and resulted in a significant improvement in goodness-offit values $\left(f^{\prime}\right)$. Therefore, the second-shell FT feature observed at $3.34 \AA$ is consistent with a dinuclear $\mathrm{Zn}(\mathrm{II})$ active site in DapE. Although fits for [Zn_(DapE)] that include a Zn-Zn interaction improve the $f^{\prime}$ 'values slightly, the Debye-Waller factor value for this interaction is significantly higher than for the same interaction in [ZnZn(DapE)] (cf. Fits 2,4; Table 1).
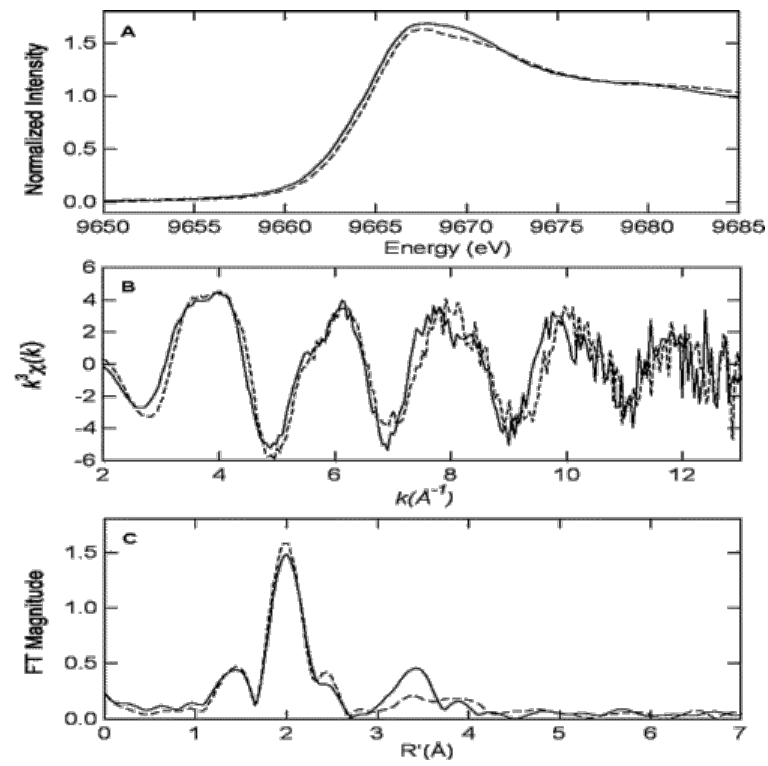

Figure 1 Zn K-edge X-ray absorption spectra for [Zn_(DapE)] (dashed) and [ZnZn(DapE)] (solid). (A) Zn edge spectra; (B) $k^{3}$-weighted Zn EXAFS; (C) Fourier transforms over $k=2-13 \AA^{-1}$, with $S$ phase correction. 

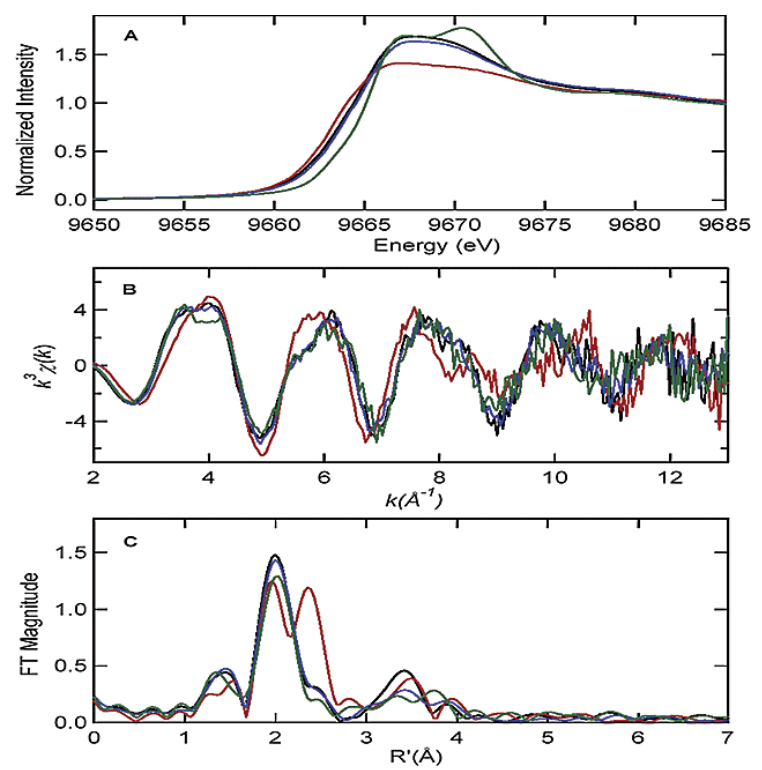

Figure $2 \mathrm{Zn} \mathrm{K-edge} \mathrm{X-ray} \mathrm{absorption} \mathrm{spectra} \mathrm{of} \mathrm{[ZnZn(DapE)]} \mathrm{in} \mathrm{the} \mathrm{absence} \mathrm{(black)} \mathrm{and} \mathrm{presence} \mathrm{of} \mathrm{CEPA} \mathrm{(blue)}$ or MSPA (red) and [ZnZn(APP)] in the presence of LPA (green). (A) Zn edge spectra; (B) $k^{3}$-weighted Zn EXAFS; (C) Fourier transforms over $k=2-13 \AA^{-1}$, with $S$ phase correction.

Table 1. Curve-Fitting Results for Zn EXAFS ${ }^{a}$ of DapE Enzymes

\begin{tabular}{|c|c|c|c|c|c|c|}
\hline sample filename ( $k$ range) $\Delta k^{3} \chi$ & fit & shell & $R_{\mathrm{as}}(\AA)$ & $\sigma_{\mathrm{as}}^{2}\left(\AA^{2}\right)$ & $\Delta E_{0}(\mathrm{eV})$ & $f^{\prime b}$ \\
\hline [Zn_(DapE)] & 1 & $\mathrm{Zn}-\mathrm{O}_{5}$ & 1.96 & 0.0062 & -9.11 & 0.104 \\
\hline $\operatorname{ZDZOA}\left(2-13 \AA^{-1}\right)$ & 2 & $\mathrm{Zn}-\mathrm{O}_{5}$ & 1.96 & 0.0062 & -9.30 & 0.100 \\
\hline$\Delta k^{3} \chi=10.23$ & & $Z n-Z n$ & 3.30 & 0.0117 & & \\
\hline [ZnZn(DapE)] & 3 & $\mathrm{Zn}-\mathrm{O}_{5}$ & 1.98 & 0.0065 & -9.01 & 0.111 \\
\hline ZDZOA $\left(2-13 \AA^{-1}\right)$ & 4 & $\mathrm{Zn}-\mathrm{O}_{5}$ & 1.98 & 0.0064 & -9.41 & 0.093 \\
\hline$\Delta k^{3} \chi=10.44$ & & $Z n-Z n$ & 3.34 & 0.0051 & & \\
\hline [ZnZn(AAP)] & 5 & $\mathrm{Zn}-\mathrm{O}_{5}$ & 1.99 & 0.0084 & -8.71 & 0.094 \\
\hline ZAZZA $\left(2-13 \AA^{-1}\right)$ & 6 & $\mathrm{Zn}-\mathrm{O}_{5}$ & 1.99 & 0.0084 & -9.12 & 0.089 \\
\hline$\Delta k^{3} \chi=9.47$ & & $Z n-Z n$ & 3.27 & 0.0099 & & \\
\hline [ZnZn(DapE)]-CEPA & 7 & $\mathrm{Zn}-\mathrm{O}_{5}$ & 1.99 & 0.0069 & -8.00 & 0.096 \\
\hline ZDZOA $\left(2-13 \AA^{-1}\right)$ & 8 & $\mathrm{Zn}-\mathrm{O}_{5}$ & 1.99 & 0.0085 & -8.42 & 0.088 \\
\hline$\Delta k^{3} \chi=9.89$ & & $Z n-Z n$ & 3.34 & 0.0083 & & \\
\hline [ZnZn(AAP)]-LPA & 9 & $\mathrm{Zn}-\mathrm{O}_{5}$ & 1.99 & 0.0078 & -8.22 & 0.108 \\
\hline ZAZPA $\left(2-13 \AA^{-1}\right)$ & 10 & $\mathrm{Zn}-\mathrm{O}_{5}$ & 1.99 & 0.0077 & -8.56 & 0.103 \\
\hline \multirow[t]{3}{*}{$\Delta k^{3} \chi=9.91$} & & $Z n-Z n$ & 3.30 & 0.0112 & & \\
\hline & 11 & $\mathrm{Zn}-\mathrm{O}_{5}$ & 1.99 & 0.0077 & -8.49 & 0.103 \\
\hline & & $Z n-Z n$ & 3.80 & 0.0072 & & \\
\hline [ZnZn(DapE)]-MSPA & 12 & $\mathrm{Zn}-\mathrm{O}_{5}$ & 1.99 & 0.0079 & -3.03 & 0.109 \\
\hline ZDZTA $\left(2-13 \AA^{-1}\right)$ & 13 & $\mathrm{Zn}-\mathrm{O}_{5}$ & 1.99 & 0.0050 & -6.24 & 0.097 \\
\hline \multirow[t]{2}{*}{$\Delta k^{3} \chi=9.98$} & & $\mathrm{Zn}-\mathrm{S}$ & 2.30 & 0.0089 & & \\
\hline & & $Z n-Z n$ & 3.64 & 0.0083 & & \\
\hline
\end{tabular}

${ }^{a}$ Shell is the chemical unit defined for the multiple scattering calculation, the number of scatterers per metal denoted by the subscript. $R_{\text {as }}$ is the metal-scatterer distance. $\sigma_{\text {as }}{ }^{2}$ is a mean square deviation in $R_{\text {as. }} \Delta E_{0}$ is the shift in $E_{0}$ for the theoretical scattering functions. ${ }^{b} f^{\prime}$ is a normalized error ( $\chi$-squared): $f^{\prime}=$ $\left\{\sum_{i}\left[k^{3}(-)\right]^{2} / N\right\}^{1 / 2} /\left[\left(k^{3} \chi^{\text {obs }}\right)_{\max }-\left(k^{3} \chi^{\text {obs }}\right)_{\min }\right]$. 
Sequence alignments of several DapE genes with those of the crystallographically characterized aminopeptidase from Aeromonas proteolytica (AAP) and the carboxypeptidase $\mathrm{G}_{2}$ from Pseudomonas sp. strain RS-16 (CPG $)$, indicate that all of the amino acids that function as metal ligands in $A A P$ and $C G_{2}$ are strictly conserved in DapEs. ${ }^{11,14-16}$ The $X$-ray crystal structures of both $\mathrm{CPG}_{2}$ and AAP reveal a ( $\mu$-aquo)( $\mu$-carboxylato)dizinc(II) active site with one terminal carboxylate and histidine residue bound to each metal ion. ${ }^{15,16}$ Both $\mathrm{Zn}(\mathrm{II})$ ions in AAP and $\mathrm{CPG}_{2}$ reside in a distorted tetrahedral coordination geometry, with a $\mathrm{Zn}-\mathrm{Zn}$ distance of 3.5 and $3.3 \AA$ for AAP and $\mathrm{CPG}_{2}$, respectively. ${ }^{15,16}$ EXAFS data obtained for $[\mathrm{ZnZn}(\mathrm{AAP})]$ also contain a feature at $3.27 \AA$ that can be modeled as a $\mathrm{Zn}-\mathrm{Zn}$ vector (Fit 6; Table 1). Combination of these data indicates that DapE enzymes contain dinuclear $\mathrm{Zn}(\mathrm{II})$ active sites. In addition, on the basis of the first shell fits of [ZnZn(AAP)] and [ZnZn(DapE)], we propose that one carboxylate and one histidine residue resides at each $\mathrm{Zn}$ (II) site in DapE and that the two $\mathrm{Zn}$ (II) ions are bridged by one carboxylate residue and a water molecule.

The first step in inhibitor design for DapE enzymes requires an understanding of how inhibitors bind to the active site. Therefore, we recorded the Zn K-edge EXAFS spectra of [ZnZn(DapE)] in the presence of the competitive inhibitors 2-carboxyethylphosphonic acid (CEPA) and 5-mercaptopentanoic acid (MSPA) (Figure 2). The EXAFS data for [ZnZn(DapE)]-CEPA indicate that the average coordination number of each $\mathrm{Zn}(\mathrm{II})$ ion remains five and the $\mathrm{Zn}-\mathrm{Zn}$ distance remains 3.34 (Fits 7,8; Table 1). An increase in the M-M distance of [ZnZn(AAP)], from 3.5 to $3.9 \AA$, is observed upon the addition of the transition-state analogue inhibitor l-leucine-phosphonic acid (LPA) which contains a similar ligating group to CEPA. ${ }^{17}$ The $X$-ray crystal structure and EXAFS data for [ZnZn(AAP)]-LPA, reveal that the bridging water molecule is displaced by LPA resulting in an $\eta-1,2-\mu-$ phosphonate bridge and an increase in the $Z n-Z n$ distance of $0.4 \AA$ (Fit 11; Table 1). The fact that the $Z n-Z n$ distance does not change upon CEPA binding to [ZnZn(DapE)] suggests an $\eta-1$ - $\mu$-phosphonate bridge exists, similar to the binding mode of LPA to the leucine aminopeptidase from bovine lens. ${ }^{17,18}$ On the other hand, MSPA binding to [ZnZn(DapE)] has a marked effect on the Zn K-edge, suggesting that the average electronic environment of the dinuclear $\mathrm{Zn}$ (II) site has changed significantly (Figure 2). The observed shift to lower energy in the absorption edge position is indicative of a net increase in electron density at the dinuclear Zn(II) site, consistent with a sulfur ligand. In addition, the EXAFS data for [ZnZn(DapE)]-MSPA reveal a new feature at $2.3 \AA$ that is highly characteristic of a direct zinc-sulfur interaction (Fits 12,13; Table 1). Moreover, the M-M distance is lengthened from 3.34 to $3.64 \AA$. These data indicate that the thiol group of MSPA binds to one or more of the $\mathrm{Zn}(\mathrm{II})$ ions in the active site of DapE.

In conclusion, DapEs are potential molecular targets for a new and novel class of antibiotics. The EXAFS data presented herein provide the first structural information for any DapE enzyme and also establish the binding modes of phosphonate- and thiolate-containing inhibitors. In addition, the structural data obtained for CEPA bound to [ZnZn(DapE)] provide the first glimpse at the transition state of the hydrolysis reaction catalyzed by DapE. Since most pharmaceuticals target the transition state of enzymatic reactions, the structural aspects of [ZnZn(DapE)]-CEPA are particularly important for the rational design of new potent inhibitors of DapE enzymes.

\section{Supporting Information Available}

Experimental details (PDF). This material is available free of charge via the Internet at http://pubs.acs.org.

\section{Terms \& Conditions}

Electronic Supporting Information files are available without a subscription to ACS Web Editions. The American Chemical Society holds a copyright ownership interest in any copyrightable Supporting Information. Files available from the ACS website may be downloaded for personal use only. Users are not otherwise permitted to reproduce, republish, redistribute, or sell any Supporting Information from the ACS website, either in whole or in part, in either machine-readable form or any other form without permission from the American Chemical 
Society. For permission to reproduce, republish and redistribute this material, requesters must process their own requests via the RightsLink permission system. Information about how to use the RightsLink permission system can be found at http://pubs.acs.org/page/copyright/permissions.html.

\section{Acknowledgment}

This work was supported by the National Institutes of Health (GM-42025, R.A.S.) and the National Science Foundation (CHE-0240810, R.C.H.). The E. coli BL21(DE3) strain expressing the recombinant $H$. influenzae dapEencoded desuccinylase was provided by Professor John S. Blanchard (Albert Einstein College of Medicine, supported by Al-33696).

\section{References}

1 Henery, C. M. Chem. Eng. News2000, 41-58.

2 Snider, D. E.; Raviglione, M.; Kochi, A. In Global Burden of Tuberculosis; Bloom, B. R., Ed.; ASM Press: Washington, DC, 1994; pp 3-11.

3 Prevention. Centers for Disease Control and Prevention: Washington, DC. MMWR Morb. Mortal. Wkly Rep.1995, 44, 1-13.

4 Howe, R. A.; Bowker, K. E.; Walsh, T. R.; Feest, T. G.; MacGowan, A. P. Lancet1997, 351, 601-602.

5 Levy, S. B. Sci. Am.1998, 278, 46-53.

6 Chin, J. New Sci.1996, 152, 32-35.

7 Scapin, G.; Blanchard, J. S. Adv. Enzymol.1998, 72, 279-325.

8 Born, T. L.; Blanchard, J. S. Curr. Opin. Chem. Biol.1999, 3, 607-613.

9 Girodeau, J.-M.; Agouridas, C.; Masson, M.; R., P.; LeGoffic, F. J. Med. Chem.1986, 29, 1023-1030.

10 Velasco, A. M.; Leguina, J. I.; Lazcano, A. J. Mol. Evol.2002, 55, 445-459.

11 Born, T. L.; Zheng, R.; Blanchard, J. S. Biochemistry1998, 37, 10478-10487.

12 Karita, M.; Etterbeek, M. L.; Forsyth, M. H.; Tummuru, M. R.; Blaser, M. J. Infect. Immun.1997, 65, 4158-4164. 13 Pavelka, M. S.; Jacobs, W. R. J. Bacteriol.1996, 178, 6496-6507.

14 Makarova, K. S.; Grishin, N. V. J. Mol. Biol.1999, 292, 11-17.

15 Chevrier, B.; Schalk, C.; D'Orchymont, H.; Rondeau, J.-M.; Moras, D.; Tarnus, C. Structure1994, 2, 283-291.

16 Rowsell, S.; Pauptit, R. A.; Tucker, A. D.; Melton, R. G.; Blow, D. M.; Brick, P. Structure1997, 5, 337-347.

17 Stamper, C.; Bennett, B.; Edwards, T.; Holz, R. C.; Ringe, D.; Petsko, G. Biochemistry2001, 40, 7034-7046.

18 Sträter, N.; Lipscomb, W. N. Biochemisty1995, 34, 9200-9210. 